P093 DISTINCT AND OVERLAPPING ACTIVITIES OF IL-17A AND TNF ON THE EXPRESSION OF PROINFLAMMATORY CYTOKINES AND MMPS IN PSORIATIC ARTHRITIS: RATIONALE FOR ANTI-IL-17A/ANTI-TNFALPHA COMBINATION THERAPY?

${ }^{1} \mathrm{X} \mathrm{Xu}^{*},{ }^{1} \mathrm{~N}$ Davelaar, ${ }^{1} \mathrm{~A}-\mathrm{M}$ Otten-Mus, ${ }^{1} \mathrm{P}$ Asmawidjaja, ${ }^{1} \mathrm{~J}$ Hazes, ${ }^{2} \mathrm{D}$ Baeten, ${ }^{1} \mathrm{M}$ Vis, ${ }^{1} \mathrm{R}$ Bisoendial, ${ }^{1} \mathrm{E}$ Lubberts. ${ }^{1}$ Erasmus MC Rotterdam, Rotterdam; ${ }^{2}$ Academic medical centre, Amsterdam, Netherlands

10.1136/annrheumdis-2018-EWRR2018.109

Introduction TNF and IL-17A are proinflammatory cytokines critically involved in the pathogenesis of psoriatic arthritis (PsA). Currently, targeting TNF is the first choice of a biologic disease-modifying antirheumatic drug (bDMARD) in PsA. However, up to $30 \%$ of patients receiving anti-TNF monotherapy fail to respond and require switching to a second TNF-inhibitor or bDMARD with different modes of action. Strategies targeted at neutralising IL-17A have shown beneficial effects on skin, enthesitis, dactylitis and joint inflammation.

Objectives We explore the effect of neutralising IL-17A versus anti-TNF on the expression of proinflammatory cytokines and metalloproteinases (MMPs) and whether dual-therapy targeting TNF and IL-17A may have superior activity than treatment with either agent alone.

Methods An allogeneic co-culture system was used comprising synovial fluid $\mathrm{T}$ helper (Th) memory cells and synovial fibroblasts (SF), derived from patients with active PsA. Anti-CD3 and CD28 stimulation was used during culture, and anti-IL17A antibody (Secukinumab), anti-TNF $\alpha$ antibody (Adalimumab), or the combination were added. PsA unstimulated synovial Th memory cells co-cultured with PsA SF were included as a control group as well as an isotype antibody control group. After 72 hours, supernatants were harvested for ELISA and cells were lysed for qPCR analysis.

Results Anti-TNF Ab treatment had no effect on IL-17A levels and neutralisation of IL-17A did not influence the TNF production in co-culture system. Both anti-TNF and anti-IL-17A single treatment significantly inhibited the production of IL-8 and reduced the mRNA expression of IL-1 $\beta$. Interestingly, neutralising IL-17A resulted in a significant suppression of IL6 level which was not reduced by anti-TNF. Anti-TNF inhibited the production of MMP-3 and only the combination of anti-TNF and anti-IL-17A resulted in a significant suppression of MMP-1 levels and MMP-9 mRNA expression. MMP-13 mRNA expression was significantly suppressed by anti-TNF but not by anti-IL-17A. However, neutralising both showed a significant improvement in downregulating MMP-13 mRNA expression compared to single treatment. Moreover, anti-IL$17 \mathrm{~A}$ reduced RANK mRNA expression significantly compared to anti-TNF, but no additive effect was noted for combination blocking. Interestingly, only neutralising both IL-17A and TNF significantly reduced the mRNA expression of RANKL. OPG mRNA expression was not influenced by either treatment or both.

Conclusions Neutralising IL-17A or TNF in the PsA synovial $\mathrm{T}$ cell-SF co-culture system resulted in overlapping but also distinct effects on proinflammatory cytokine expression. TNF inhibition markedly suppress different MMPs with mostly an additional effect upon neutralisation of IL17A. Neutralisation both IL-17A and TNF is needed to downregulate RANKL expression which changes the RANKL/OPG balance. Together, these data suggest that dual therapy targeting IL-17A and
TNF may be superior in their activity to protect against erosive arthropathy in PsA than treatment with either agent alone.

Disclosure of interest X. Xu: None declared, N. Davelaar: None declared, A.-M. Otten-Mus: None declared, P. Asmawidjaja: None declared, J. Hazes: None declared, D. Baeten: None declared, M. Vis: None declared, R. Bisoendial: None declared, E. Lubberts Grant/research support from: Novartis

\section{P094 ASSESSMENT OF INTRACRANIAL VESSELS AND VASCULAR LESIONS IN RHEUMATOID ARTHRITIS. A DETAILED TRANSCRANIAL DOPPLER, CAROTID ULTRASOUND AND BRAIN MRI STUDY}

\begin{abstract}
${ }^{1} \mathrm{Z}$ Kardos, ${ }^{2} \mathrm{C}$ Oláh, ${ }^{3} \mathrm{M}$ Sepsi, ${ }^{4} \mathrm{~A}$ Sas, ${ }^{3} \mathrm{~L}$ Kostyál, ${ }^{5} \mathrm{H}$ Bhattoa, ${ }^{1} \mathrm{~K}$ Hodosi, ${ }^{1} \mathrm{~A}$ Domián, ${ }^{6} \mathrm{G}$ Kerekes, ${ }^{7} \mathrm{~L}$ Tamási, ${ }^{4} \mathrm{~A}$ Valikovics, ${ }^{8} \mathrm{D}$ Bereczki, ${ }^{1} \mathrm{Z}$ Szekanecz ${ }^{*}$. ${ }^{1}$ Department of Rheumatology; ${ }^{2}$ Department of Neurosurgery; ${ }^{3}$ Department of Radiology; ${ }^{4}$ Department of Neurology, Borsod County Teaching Hospital, Miskolc; ${ }^{5}$ Department of Laboratory Medicine; ${ }^{6}$ Department of Angiology, University of Debrecen, Faculty of Medicine; ${ }^{7}$ Department of Rheumatology, Borsod County Teaching Hospital, Debrecen; ${ }^{8}$ Department of Neurology, Semmelweis University, Budapest, Hungary
\end{abstract}

\subsection{6/annrheumdis-2018-EWRR2018.110}

Introduction Stroke has been associated with rheumatoid arthritis (RA). Vascular physiology should be assessed in the preclinical vascular state. Assessment of intracranial vessels includes transcranial doppler (TCD). TCD performance requires intact temporal acoustic windows (TAW). Failure of TAW (TAWF) is present in $8 \%-20 \%$ of people. This may be due to pathological bone metabolism.

Objectives We assessed RA patients and healthy controls by transcranial Doppler (TCD), carotid ultrasonography and brain MRI. We wished to determine preclinical pathophysiological changes in the cerebral vasculature.

Methods TAWF, temporal bone thickness and texture were determined by ultrasound and CT. BMD and T-scores of multiple bones were determined by DEXA. Several bone biomarkers were assessed by ELISA. Altogether 63 female RA patients and 60 age-matched controls underwent TCD assessment of the medium cerebral (MCA), basilar and vertebral arteries. Pulsatility(PI), resistance(RI) indices and circulatory reserve capacity (CRC) were determined. The presence of carotid plaques and intima-media thickness (cIMT) were also determined. Intracerebral vascular lesions were investigated by brain MRI. RA subsets include MTX- and biologic-treated patients.

Results In RA, the right and left TAW were undetectable in $35 \%$ and $53 \%$ of patients, respectively. In controls, TAWF was only present in $20 \%$ of subjects. Temporal bone was thicker, and texture was more heterogeneous in RA patients was controls $(p<0.05)$. These TAW features were generally more common on the left side. There was close association between TAWF, bone thickness and texture $(p<0.05)$. These TAW parameters all correlated with age, however TAW failure and texture also correlated with serum osteoprotegerin. TAW bone thickness inversely correlated with hip BMD $(\mathrm{p}<0.05)$.

Conclusions TAWF, thicker and heterogeneous temporal bones were associated with RA. These features have been associated with bone loss and OPG production. Bone loss seen in RA may result in OPG release and stimulation of bone formation around TAW. This may be the first study to show increased distal MCA and basilar artery occlusion in RA as determined by TCD. RA patients also exert CRC defect. We also 